\title{
Reductive evolution and the loss of PDC/PAS domains from the genus Staphylococcus
}

\author{
Neethu Shah', Rosmarie Gaupp ${ }^{2}$, Hideaki Moriyama ${ }^{3}$, Kent M Eskridge ${ }^{4}$, Etsuko N Moriyama ${ }^{5^{*}}$ \\ and Greg A Somerville $2^{*}$
}

\begin{abstract}
Background: The Per-Arnt-Sim (PAS) domain represents a ubiquitous structural fold that is involved in bacterial sensing and adaptation systems, including several virulence related functions. Although PAS domains and the subclass of PhoQ-DcuS-CitA (PDC) domains have a common structure, there is limited amino acid sequence similarity. To gain greater insight into the evolution of PDC/PAS domains present in the bacterial kingdom and staphylococci in specific, the PDC/PAS domains from the genomic sequences of 48 bacteria, representing 5 phyla, were identified using the sensitive search method based on HMM-to-HMM comparisons (HHblits).

Results: A total of 1,007 PAS domains and 686 PDC domains distributed over 1,174 proteins were identified. For 28 Gram-positive bacteria, the distribution, organization, and molecular evolution of PDC/PAS domains were analyzed in greater detail, with a special emphasis on the genus Staphylococcus. Compared to other bacteria the staphylococci have relatively fewer proteins (6-9) containing PDC/PAS domains. As a general rule, the staphylococcal genomes examined in this study contain a core group of seven PDC/PAS domain-containing proteins consisting of WalK, SrrB, PhoR, ArlS, HssS, NreB, and GdpP. The exceptions to this rule are: 1) S. saprophyticus lacks the core NreB protein; 2) S. carnosus has two additional PAS domain containing proteins; 3) S. epidermidis, S. aureus, and S. pseudintermedius have an additional protein with two PDC domains that is predicted to code for a sensor histidine kinase; 4) S. lugdunensis has an additional PDC containing protein predicted to be a sensor histidine kinase.

Conclusions: This comprehensive analysis demonstrates that variation in PDC/PAS domains among bacteria has limited correlations to the genome size or pathogenicity; however, our analysis established that bacteria having a motile phase in their life cycle have significantly more PDC/PAS-containing proteins. In addition, our analysis revealed a tremendous amount of variation in the number of PDC/PAS-containing proteins within genera. This variation extended to the Staphylococcus genus, which had between 6 and 9 PDC/PAS proteins and some of these appear to be previously undescribed signaling proteins. This latter point is important because most staphylococcal proteins that contain PDC/PAS domains regulate virulence factor synthesis or antibiotic resistance.
\end{abstract}

Keywords: Staphylococcus, PAS domain, Molecular evolution, Phylogeny

\section{Background}

Basic cellular functions (e.g., DNA replication and transcription) are regulated in response to environmental and nutritional signals. For this reason, sensing and transduction of these extracellular and intracellular signals is of critical importance for survival. Many of the

\footnotetext{
*Correspondence: emoriyama2@unl.edu; gsomerville3@unl.edu

${ }^{5}$ School of Biological Sciences and Center for Plant Science Innovation, University of Nebraska, Lincoln, NE 68588-0118, USA

${ }^{2} \mathrm{School}$ of Veterinary Medicine and Biomedical Sciences, University of Nebraska, Lincoln, NE 68583-0905, USA

Full list of author information is available at the end of the article
}

signaling and regulatory proteins involved in responding to environmental cues have a modular composition of specialized domains $[1,2]$. One of these specialized domains is the Per-Arnt-Sim (PAS) domain, which is distributed throughout all genera of life. PAS domains are identified by particular patterns of secondary structures and they have important roles as sensory modules for environmental and nutritional signals, such as voltage, gases, light, metabolites or redox potential [3-5]. In addition to regulating physiological processes, several PAS domain containing proteins are involved in regulating virulence determinants in pathogenic bacteria [6-10].

\section{Biomed Central}


The wide-distribution of PAS domains, the diversity of signals they transduce, and the array of regulatory pathways in which they are involved are reflected in their extremely divergent amino acid sequences [11-13]. Our interest in PAS domain-containing proteins is due to their involvement in sensing and responding to changes in concentrations of dicarboxylic acids [14].

Bacterial survival depends on their ability to regulate transcription of genes in response to changing environmental and nutritional conditions. In pathogenic bacteria, the response to changes in nutritional or environmental conditions often includes activating transcription of virulence genes [15]. The genus Staphylococcus includes both pathogenic and non-pathogenic species that live in diverse environmental conditions such as the anterior nares (i.e., S. aureus), the skin (i.e., S. epidermidis), and in dry sausages (i.e., S. carnosus) [16]. The medical and economic importance of staphylococci has generated considerable interest in how these bacteria "sense" and respond to changes in their environment. In part, the medical interest stems from a desire to control staphylococcal infections, while the commercial interests involve understanding the role of primary and secondary metabolites in flavoring fermented meats. This interest in environmental sensing and the importance of PAS domains in regulating responses to environmental and nutritional signals led us to examine eight genomes of seven staphylococcal species for the presence of PAS domains. Specifically, we wanted to identify potential dicarboxylic acid sensing proteins that could link changes in tricarboxylic acid cycle activity to transcriptional alterations that affect virulence factor synthesis [15]. To gain insight into the potential functions and evolutionary relationships of these domains, we compared the staphylococcal PAS domains to those of a diverse group (47 different species) of bacteria representing five phyla.

PAS domains are typically between 100-120 amino acids in length; however, some are larger $[4,11,17]$. The core of a PAS domain is a five stranded $\beta$-sheet, and interspersed within this core are $\alpha$-helices that provide ligand/signal specificity [4,18]. The position of $\alpha$-helices can vary depending on the cellular location; specifically, cytoplasmic PAS domains have an $\alpha-2 \beta-4 \alpha-3 \beta$ topology (Figure 1A) and extracytoplasmic PAS domains have a $3 \alpha-2 \beta-1 / 2 \alpha-3 \beta-\alpha$ topology (Figure 1B) $[13,18,19]$. These extracytoplasmic PAS domains are typified by the PhoQ,

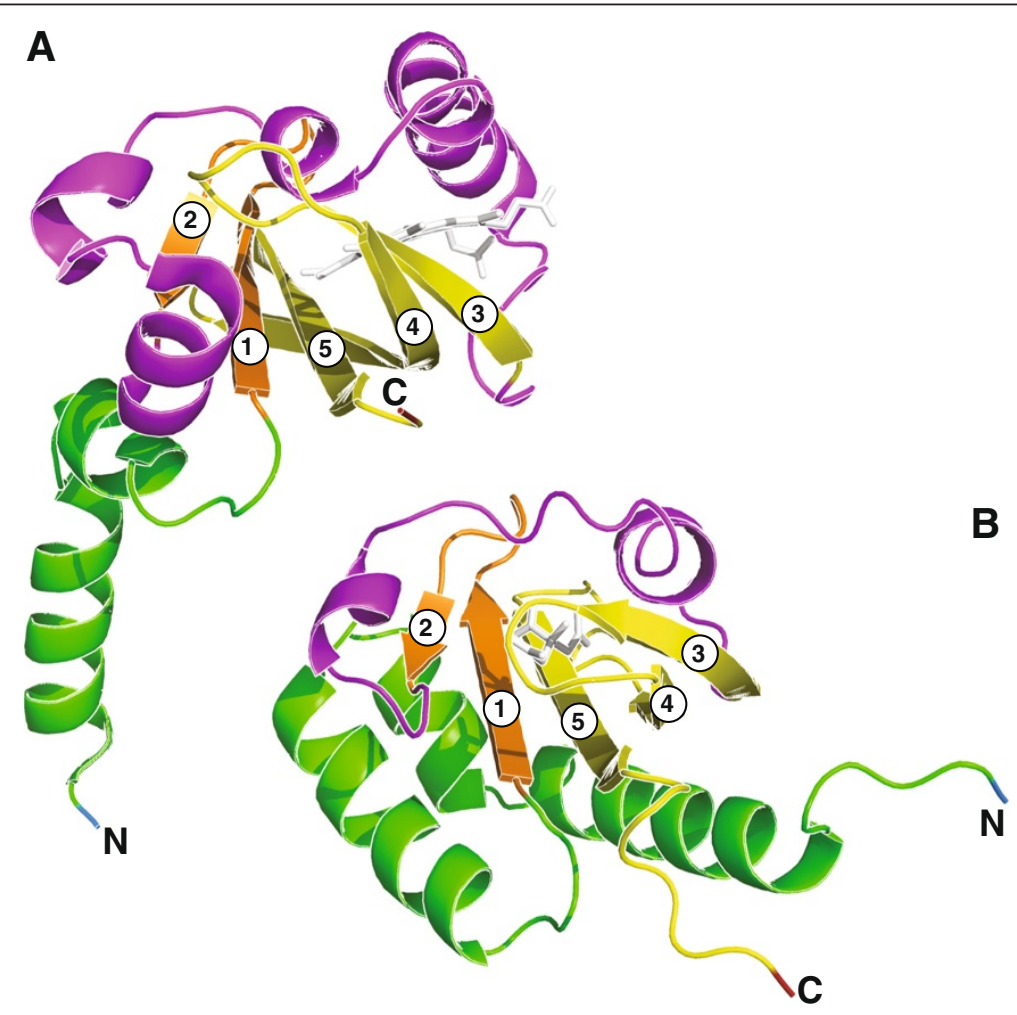

Figure 1 Three-dimensional structures of PAS (A) and PDC (B) domains. The structures are based on (A) the Rhizobium meliloti oxygen sensor FixL protein with its ligand heme (UniProt P10955: positions 122-251) (PDB: 1D06) [21], and (B) the ligand-binding domain of the Klebsiella pneumoniae sensor kinase CitA protein with its ligand citrate (UniProt P52687: positions 5-135) (PDB: 1P0Z) [22]. For both structures, the core $\beta$ strands are labeled from 1 to 5. Schematic models were generated by PyMol (Schrödinger, Portland, OR). Each region is colored as follows: the amino end with blue, the leading a-helix region with green, the first two $\beta$-strands with orange, the inter-domain a-helix region with magenta, the last three $\beta$-strands with yellow, and the carboxyl end with red. Ligands are shown in white stick models. 
DcuS, and CitA proteins, and are referred to as PDC domains [20].

PAS domains share less than $20 \%$ amino acid sequence identity $[4,11-13]$; hence, identifying PDC/PAS domain containing proteins across diverse genomes has been difficult. Taylor and Zhulin [4] used position-specific iterative BLAST (PSI-BLAST) [23] to identify 68 PAScontaining proteins from eleven bacterial genomes. Of the eleven genomes, only six were found to have PAScontaining proteins and several of these proteins included multiple PAS domains. An analysis of sixteen bacterial species using PSI-BLAST similarity search revealed a wide distribution of small-molecule binding domains, including PAS domains, in signal transducing proteins, transporters, and enzymes [1]. In a search of PAS domain containing proteins in two genomes of Cyanobacteria (i.e., Nostoc punctiforme and Anabena sp.), Narikawa et al. [24] used profile hidden Markov models (HMMs) to identify a total of 323 PAS domains. More recently, using the fold and function assignment system (FFAS) algorithm [25], Chang et al. [19] found that eleven out of thirteen sensor kinases in the Bacillus subtilis genome possessed PDC domains.

In order to expand our understanding of PDC and PAS domain distribution in bacteria and their evolutionary history, we chose to use more sensitive search methods, on a more diverse array of bacterial genomes. Specifically, we used the recently developed sensitive search method based on HMM-to-HMM comparisons (HHblits) [26]. Using this approach, we identified a total of 1,174 PDC/PAS domain containing proteins (for brevity, we call them PDC/ PAS proteins henceforth) in 48 diverse bacterial genomes, including both Gram-negative and Gram-positive bacteria. The number of PDC/PAS proteins varied across these genomes (ranging from 3 to 98 proteins) and more PAS domains $(1,007)$ were identified than PDC domains (686). Interestingly, the number of PDC/PAS proteins also varied among the eight staphylococcal genomes; as an example, Staphylococcus saprophyticus had six PDC/PAS proteins, while Staphylococcus carnosus had nine. Lastly, these data provided an opportunity to examine the evolution and function of staphylococcal PDC/PAS proteins.

\section{Results and discussion}

\section{PDC/PAS domains in evolutionarily diverse bacteria}

The importance of PAS domains in bacterial signal transduction systems and the abundance of staphylococcal genomic DNA sequences provided an excellent opportunity to analyze staphylococcal genomes for the presence of PDC and PAS domains. In order to understand the molecular evolution of staphylococcal PDC and PAS domains within the bacterial kingdom, we identified these domains from a diverse set of 48 bacterial genomes, including seven species of Staphylococcus (Table 1). Due to the availability of multiple S. aureus genomes and their medical importance, two strains (COL and USA300_TCH1516) of S. aureus were included in the analyses. The domains were classified based on the predicted secondary structure as PAS $(\alpha-$ $2 \beta-4 \alpha-3 \beta)$ or PDC $(3 \alpha-2 \beta-1 / 2 \alpha-3 \beta-\alpha)$ folds (Table 1 and found in Additional file 1: Table S1). A total of 1,693 PAS/PDC domains in 1,174 proteins were identified, of which 1,007 were PAS folds and 686 domains were PDC folds. Consistent with the ubiquitous nature of PAS domains, all bacteria examined had PAS domains. Similarly, PDC domains were found in all genomes examined except for Lactococcus lactis. As previously observed [24], the filamentous cyanobacterium Nostoc punctiforme had the greatest number of total PDC/PAS domains (169 domains distributed over 97 proteins), as well as the greatest number of PAS domains (131 domains) while Vibrio cholerae had the most PDC domains (59 domains) (Table 1). In general, smaller bacterial genomes had fewer PDC/PAS proteins, hence fewer PDC/ PAS domains, while larger genomes had more PDC/PAS proteins and more PDC/PAS domains. To determine if a correlation existed between the total number of proteins and PAS domains, we calculated a correlation coefficient for these two variables. As expected, a significant positive correlation was found between the total number of proteins and the number of PDC/PAS proteins (Figure 2; Pearson's correlation coefficient $r=0.76, p<0.0001$; Spearman's rank correlation $\rho=0.82, p<0.0001$ ).

Previously, a hypothesis was put forward that nonpathogenic bacteria have more PDC/PAS proteins than do pathogenic bacteria [1]. To test this hypothesis, we grouped bacteria based on its pathogenic status and determined if the two groups (pathogenic or not) have a significant difference in regard to the PDC/PAS protein numbers (see Table 1). The average numbers of PDC/PAS proteins were 18.9 for 30 pathogenic bacteria and 33.7 for 18 nonpathogenic bacteria. This difference was minimally significant $(p=0.048$ by $t$-test, but $p>0.1$ by non-parametric Mann-Whitney $U$ test). When we removed the effect of over-representation of Staphylococcus genomes by removing 5 of the 8 genomes, all pathogenic (see Methods), no significant difference was observed (the average number of PDC/PAS proteins for 25 pathogenic genomes was 21.2; $p>0.1$ by $t$-test and $p>0.3$ by Mann-Whitney $U$ test). Comparisons of the total protein numbers between the two pathogenicity groups had the same results. Although nonpathogenic bacteria tend to have more PAS domains, the non-significant difference suggests that acquisition and/or loss of PAS domains is largely independent of pathogenicity. In addition, these data suggest that another selectable phenotypic trait(s) is responsible for the variation in the number of PDC/PAS domains in bacteria. 
Table 1 Summary of the $\mathbf{4 8}$ bacterial genomes used in this study and the PAS/PDC domains identified

\begin{tabular}{|c|c|c|c|c|c|c|c|c|c|}
\hline Species $^{a}$ & Subspecies/strain & Accession number & Phylum & Pathogenicity $^{\mathbf{b}}$ & Motility $^{c}$ & $\begin{array}{c}\text { Total } \\
\text { proteins }^{d}\end{array}$ & $\begin{array}{l}\text { PDC/PAS } \\
\text { proteins }^{\mathrm{e}}\end{array}$ & $\begin{array}{c}\text { PAS } \\
\text { domains }^{f} \\
\end{array}$ & $\begin{array}{c}\text { PDC } \\
\text { domains } \\
\end{array}$ \\
\hline \multicolumn{10}{|l|}{ [Gram positive] } \\
\hline Bifidobacterium animalis & lactis AD011 & NC_011835.1 & Actinobacteria & 0 & 0 & 1527 & 5 & 4 & 2 \\
\hline Corynebacterium diphtheriae (Ci) & NCTC 13129 & NC_002935.2 & Actinobacteria & 1 & 0 & 2272 & 4 & 2 & 2 \\
\hline Corynebacterium glutamicum (Cg) & ATCC 13032 & NC_006958.1 & Actinobacteria & 0 & 0 & 3057 & 7 & 5 & 4 \\
\hline Micrococcus luteus (MI) & NCTC 2665 & NC_012803.1 & Actinobacteria & 0 & 0 & 2236 & 5 & 4 & 3 \\
\hline Mycobacterium leprae & TN & NC_002677.1 & Actinobacteria & 1 & 0 & 1605 & 6 & 7 & 1 \\
\hline Mycobacterium smegmatis (Ms) & MC2 155 & NC_008596.1 & Actinobacteria & 0 & 0 & 6717 & 20 & 17 & 10 \\
\hline Mycobacterium tuberculosis (Mt) & CDC1551 & NC_002755.2 & Actinobacteria & 1 & 0 & 4189 & 10 & 6 & 7 \\
\hline Streptomyces coelicolor (Stc) & $\mathrm{A} 3(2)$ & NC_003888.3 & Actinobacteria & 0 & 0 & 8154 & 51 & 48 & 18 \\
\hline Bacillus anthracis $(\mathrm{Ba})$ & CDC 684 & NC_012581.1 & Firmicutes & 1 & 0 & 5902 & 43 & 30 & 30 \\
\hline Bacillus cereus (BC) & ATCC 10987 & NC_003909.8 & Firmicutes & 1 & 1 & 5843 & 41 & 30 & 25 \\
\hline Bacillus megaterium $(\mathrm{Bm})$ & QM B1551 & NC_010010.2 & Firmicutes & 0 & 1 & 5612 & 53 & 57 & 21 \\
\hline Bacillus subtilis (Bs) & subtilis 168 & NC_000964.3 & Firmicutes & 0 & 1 & 4176 & 32 & 21 & 30 \\
\hline Clostridium acetobutylicum (Ca) & ATCC 824 & NC_003030.1 & Firmicutes & 0 & 1 & 3847 & 36 & 16 & 34 \\
\hline Clostridium botulinum & A str. ATCC 3502 & NC_009496.1 & Firmicutes & 1 & 1 & 3590 & 48 & 31 & 40 \\
\hline Clostridium difficile $(\mathrm{Cd})$ & 630 & NC_009089.1 & Firmicutes & 1 & 1 & 3749 & 49 & 49 & 26 \\
\hline Enterococcus faecalis (Efs) & V583 & NC_004668.1 & Firmicutes & 1 & 0 & 3264 & 9 & 7 & 7 \\
\hline Enterococcus faecium (Efm) & DO & NZ_AAAK00000000 & Firmicutes & 1 & 0 & 3114 & 9 & 3 & 10 \\
\hline Lactobacillus casei (LC) & ATCC 334 & NC_008526.1 & Firmicutes & 0 & 0 & 2768 & 8 & 6 & 4 \\
\hline Lactococcus lactis (LI) & cremoris MG1363 & NC_009004.1 & Firmicutes & 0 & 0 & 2434 & 5 & 5 & 0 \\
\hline Listeria monocytogenes $(\mathrm{Lm})$ & EGD-e & NP_463535.1 & Firmicutes & 1 & 1 & 2846 & 12 & 9 & 7 \\
\hline Macrococcus caseolyticus (Mc) & JCSC5402 & NC_011999.1 & Firmicutes & 0 & 0 & 2052 & 7 & 6 & 4 \\
\hline Staphylococcus aureus (SaU) & $\begin{array}{l}\text { aureus } \\
\text { USA300_TCH1516 }\end{array}$ & NC_010079.1 & Firmicutes & 1 & 0 & 2693 & 8 & 5 & 7 \\
\hline Staphylococcus aureus (SaC) & aureus COL & NC_002951.2 & Firmicutes & 1 & 0 & 2612 & 8 & 5 & 7 \\
\hline Staphylococcus carnosus (Sc) & carnosus TM300 & NC_012121.1 & Firmicutes & 0 & 0 & 2461 & 9 & 8 & 5 \\
\hline Staphylococcus epidermidis (Se) & ATCC 12228 & NC_004461.1 & Firmicutes & 1 & 0 & 2416 & 8 & 5 & 7 \\
\hline Staphylococcus haemolyticus (Sh) & JCSC1435 & NC_007168.1 & Firmicutes & 1 & 0 & 2676 & 7 & 5 & $4^{h}$ \\
\hline Staphylococcus lugdunensis (SI) & HKU09-01 & CP_001837.1 & Firmicutes & 1 & 0 & 2490 & 7 & 5 & 5 \\
\hline Staphylococcus pseudintermedius (Sp) & HKU10-03 & NC_014925.1 & Firmicutes & 1 & 0 & 2450 & 8 & 5 & 7 \\
\hline Staphylococcus saprophyticus (Ss) & $\begin{array}{l}\text { saprophyticus } \\
\text { ATCC15305 }\end{array}$ & NC_007350.1 & Firmicutes & 1 & 0 & 2446 & 6 & 4 & 5 \\
\hline Streptococcus agalactiae & $2603 \mathrm{~V} / \mathrm{R}$ & NC_004116.1 & Firmicutes & 1 & 0 & 2124 & 11 & 6 & 7 \\
\hline Streptococcus pneumoniae (Spn) & D39 & NC_008533.1 & Firmicutes & 1 & 0 & 1914 & 8 & 5 & 4 \\
\hline
\end{tabular}


Table 1 Summary of the $\mathbf{4 8}$ bacterial genomes used in this study and the PAS/PDC domains identified (Continued)

\begin{tabular}{|c|c|c|c|c|c|c|c|c|c|}
\hline Streptococcus pyogenes (Spy) & MGAS10270 & NC_008022.1 & Firmicutes & 1 & 0 & 1986 & 11 & 4 & 10 \\
\hline \multicolumn{10}{|l|}{ [Gram negative] } \\
\hline Nostoc punctiforme & PCC 73102 & NC_010628.1 & Cyanobacteria & 0 & 1 & 6689 & 97 & 131 & 38 \\
\hline Synechococcus sp & CC9311 & NC_008319.1 & Cyanobacteria & 0 & 1 & 2892 & 12 & 8 & 6 \\
\hline Agrobacterium tumefaciens & C58 & NC_003062.2 & Proteobacteria & 0 & 1 & 5355 & 63 & 61 & 27 \\
\hline Bartonella henselae & Houston-1 & NC_005956.1 & Proteobacteria & 1 & 0 & 1488 & 8 & 10 & 2 \\
\hline Bradyrhizobium sp & BTAi1 & NC_009485.1 & Proteobacteria & 0 & 1 & 7621 & 98 & 103 & 51 \\
\hline Escherichia coli (Ec) & K-12 substr. MG1655 & NC_000913.2 & Proteobacteria & 0 & 1 & 4146 & 32 & 24 & 18 \\
\hline Helicobacter pylori & 83 & CP002605.1 & Proteobacteria & 1 & 1 & 1609 & 5 & 1 & 7 \\
\hline Klebsiella pneumoniae (Kp) & 342 & NC_011283.1 & Proteobacteria & 1 & 0 & 5768 & 33 & 24 & 18 \\
\hline Neisseria meningitidis & MC58 & NC_003112.1 & Proteobacteria & 1 & 0 & 2063 & 3 & 2 & 1 \\
\hline Pseudomonas aeruginosa & PAO1 & NC_002516.1 & Proteobacteria & 1 & 1 & 5571 & 70 & 71 & 35 \\
\hline Salmonella enterica & arizonae serovar & NC_010067.1 & Proteobacteria & 1 & 1 & 4500 & 21 & 12 & 13 \\
\hline Shigella flexneri & 2a str. $2457 \mathrm{~T}$ & NC_004741.1 & Proteobacteria & 1 & 0 & 4060 & 18 & 11 & 9 \\
\hline Vibrio cholerae & MJ-1236 & NC_012668.1 & Proteobacteria & 1 & 1 & 3772 & 71 & 42 & 59 \\
\hline Yersinia pestis & CO92 & NC_003143.1 & Proteobacteria & 1 & 1 & 4066 & 20 & 10 & 13 \\
\hline Leptospira biflexa & serovar Patoc & СР000788.1 & Spirochaetes & 0 & 1 & 3726 & 67 & 76 & 30 \\
\hline Treponema pallidum & pallidum SS14 & CP000805.1 & Spirochaetes & 1 & 1 & 1028 & 5 & 1 & 6 \\
\hline
\end{tabular}

${ }^{a}$ For those included in our phylogenetic analysis, abbreviations for species names are shown in parentheses.

${ }^{\mathrm{b}} 1$ : pathogenic, 0: non-pathogenic.

1: motile, 0: non-motile.

${ }^{\mathrm{d}}$ Total number of proteins in the genome.

${ }^{\mathrm{E}}$ Total number of PAS/PDC-containing proteins identified in the genome.

${ }^{f}$ Total number of PAS domains identified.

${ }^{9}$ Total number of PDC domains identified.

hOne of the PDC/PAS-containing proteins in Staphylococcus haemolyticus (Sh.3, YP_253148.1, PhoR) does not have the PDC domain identified by HHblits nor HHsearch, although all other Staphylococcus PhoR

homologs have clearly identified PDC domains. However, as noted in Supplementary Table S1, a very weakly conserved PDC-like region was identified in this protein based on the predicted secondary structure. This

potential PDC domain is not included in this table nor in our analysis. 


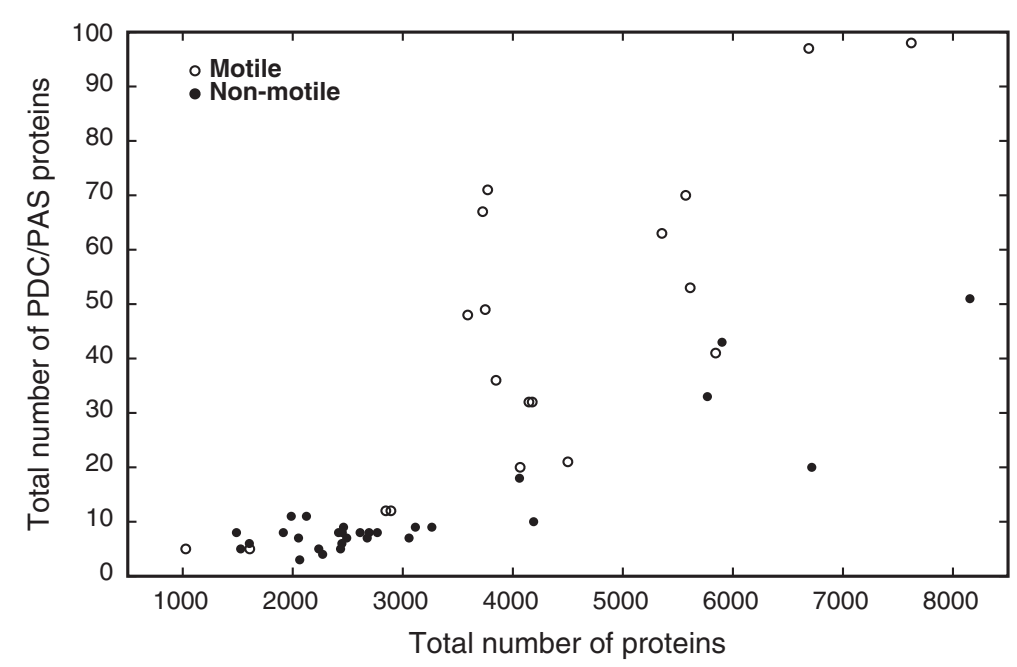

Figure 2 Correlation between the total protein numbers and the numbers of PDC/PAS proteins across $\mathbf{4 8}$ bacterial genomes. The correlation is significant either based on all 48 genomes or based on only 43 genomes (excluding 5 over-representing Staphylococcus genomes): for 48 genomes, Pearson's correlation coefficient $r=0.77(p<0.0001)$ and Spearman's rank correlation $\rho=0.83(p<0.0001)$; for 43 genomes, Pearson's correlation coefficient $r=0.76(p<0.0001)$ and Spearman's rank correlation $\rho=0.82(p<0.0001)$. Bacterial species were classified as motile or non-motile (see Table 1), and they were plotted with open and closed circles, respectively.

One of the earliest bacterial PDC/PAS proteins identified in Escherichia coli was a methyl-accepting chemotaxis protein [27]. These proteins link changes in chemotactic effector concentrations with the flagella necessary to move bacteria toward or away from that effector. The involvement of PAS domains in chemotaxis led us to examine if a correlation existed between the number of PAS domains and bacterial motility. For this analysis, a bacterium was considered motile if any part of its life-cycle involved motility (Table 1). Average numbers of PDC/PAS proteins were highly significantly different between motile (43.8 for 19 genomes) and nonmotile (12.7 for 24 genomes, excluding 5 Staphylococcus genomes) groups ( $p<0.0001$ by $t$-test). The mean number of the PDC/PAS proteins in the motile groups was approximately 4 times the mean in the non-motile group. Although variances were significantly different $(p<0.0001)$ between motility groups for the number of the PDC/PAS proteins, the test results were the same with and without the equal variance assumption. Using the non-parametric Mann-Whitney $U$ test, where normal distribution of data can be violated, median numbers of PDC/PAS proteins as well as total proteins were highly significantly different between the motility groups $(p<0.0001)$. The average total numbers of proteins (3178.9 for non-motile and 4244.1 for motile groups) were also significantly, although weakly, different $(p=$ 0.048 by $t$-test and $p=0.017$ by Mann-Whitney $U$ test). The test results were virtually the same when all 48 genomes were included in the analysis. While motility explains a substantial amount of the variation in the numbers of PDC/PAS as well as total proteins, the variation in the number of PDC/PAS proteins in bacteria must also involve other selectable phenotypes [28].

\section{PDC/PAS domain distribution in Gram-positive bacteria}

To focus the scope of this study, we limited our detailed analysis of PDC/PAS proteins to Gram-positive bacteria because they represent major health challenges in the hospital and the community [29-31]. Similar to Gramnegative bacteria, the percentage of PDC/PAS proteins in Gram-positive bacteria as a function of the total number of proteins was low $(0.97 \%$ in Gram-negative and $0.52 \%$ in Gram-positive bacteria). Also similar was that PDC/PAS proteins having a single PDC or PAS domain were the most common in both Gram-negative and Gram-positive bacteria (60.2\% and $62.1 \%$, respectively). One remarkable difference was that Gram-negative bacteria had approximately 4 times more PDC/PAS proteins that contained more than two PDC and/or PAS domains. Also consistent between Gram-negative and Gram-positive bacterial species was that the number of PDC/PAS proteins varied between genera and species (Table 1). There was also variation in the number of single and multiple PDC/PAS proteins between closely related bacteria (Figure 3 , see also Additional file 1: Table S1). For example, Streptococcus pneumoniae and Streptococcus pyogenes have 8 and 11 proteins, respectively, that contain PDC and/or PAS domains. Two of the 11 PDC/PAS proteins in S. pyogenes contain two PDC domains (i.e., 2 predicted YesM homologs: Spy.4/YP_598904.1 and Spy.5/YP_599031.1), while $S$. pneumoniae has one protein containing two PDC domains (i.e., Spn.5/YP_816074.1, predicted to be a sensor histidine kinase). Similarly, S. pyogenes has one protein containing 


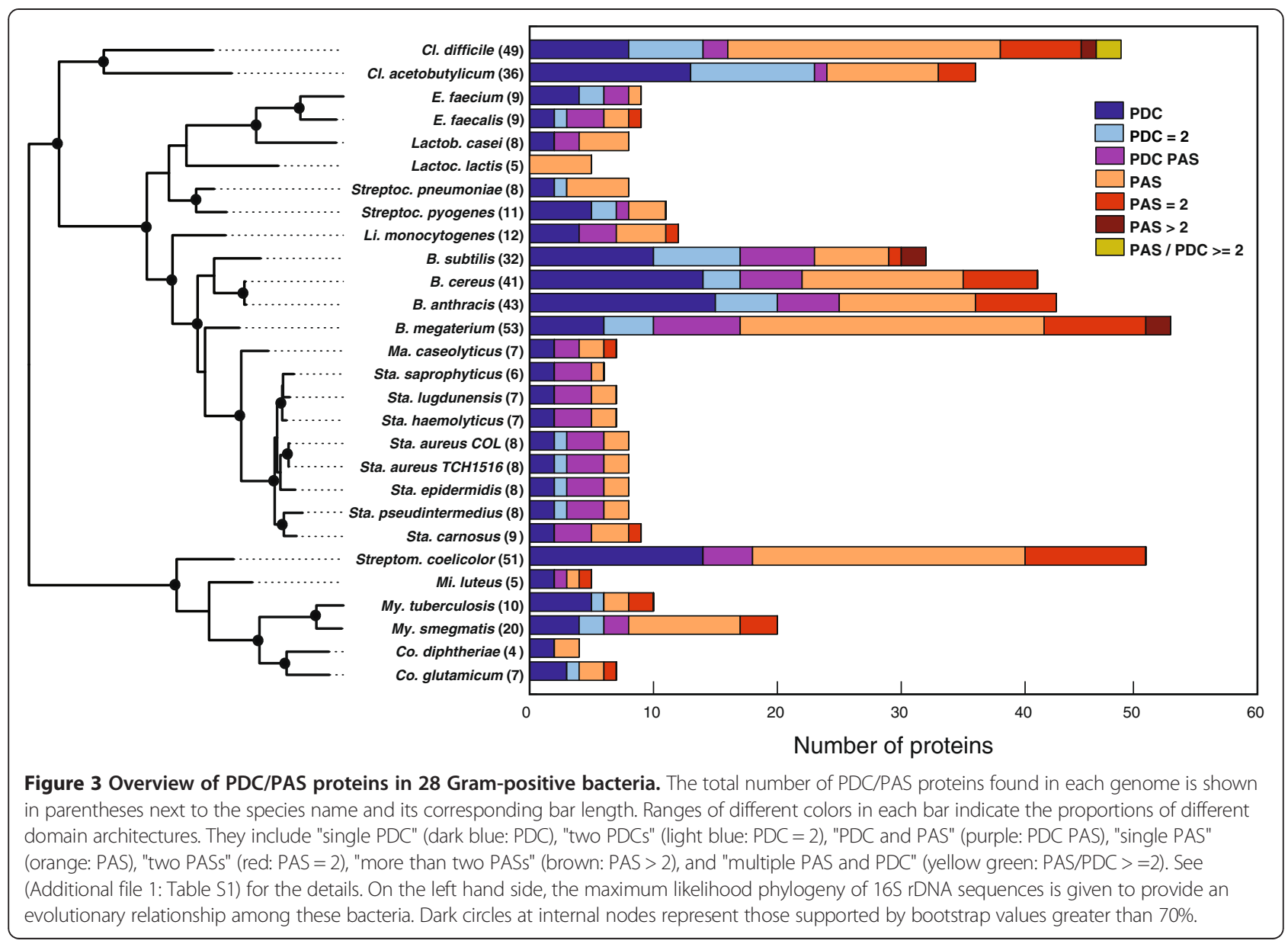

both one PDC and one PAS domains (i.e., DpiB: Spy.3/ YP_598556.1), while $S$. pneumoniae has none. Overall, our analysis found that Gram-positive bacteria tend to have fewer total PDC/PAS proteins than do Gramnegative bacteria and that Gram-positive PDC/PAS proteins infrequently have greater than 2 PDC/PAS domains. In addition, there was remarkable diversity within genera in the number of PDC/PAS proteins.

\section{Evolutionary relationships of PDC/PAS domains in Gram- positive bacteria}

In order to understand the evolutionary relationships among PAS and PDC domain sequences in bacterial genomes, we performed phylogenetic analysis using each domain sequence separately. Figure 4 shows the maximum likelihood phylogeny based on PAS domains. As indicated with very short branch lengths, PAS domains are in general highly conserved. The phylogeny also shows that PAS domains from proteins with similar predicted functions, possibly orthologous proteins, tended to cluster together. As examples, the WalK/VicK/YycG orthologs (for simplicity, we will refer to these as WalK orthologs) are part of bacterial two-component systems that regulate cell wall biosynthesis and resistance to antibiotic stress [32-34]. The WalK ortholog proteins include either one PAS or one PAS and one PDC domain ("PDC PAS" proteins shown in purple in Figure 4A). The PDC domain found in WalK is the PAS-like fold identified by Szurmant et al. as being in the extracellular loop [35]. The PAS domains for these WalK orthologs form a large clade that encompasses those from all species examined. Similarly, the "PDC PAS" containing dicarboxylate sensors (i.e., DcuS, DctS, MalK and CitS) [18] cluster together, as do the sensor kinases ResE and SrrB of the two-component anaerobiosis regulatory systems ResDE and SrrAB [36-38]. Interestingly, in the PhoR cluster, we observed that the $\mathrm{Ba}$ cillus anthracis PhoR protein contains only a single PAS domain. In other words, the PDC domain must have been lost during the evolution of the Bacillus lineage; however, their protein functions were likely retained. Another good example of PAS conservation can be found in proteins having two or more PAS domains. The two-component sensor histidine kinases, KinE, in B. subtilis (Bs.2) and B. megaterium (Bm.38) each have four PAS domains (Additional file 1: Table S1). All these PAS domains form a single cluster (shown in brown in Figure 4A), indicating 


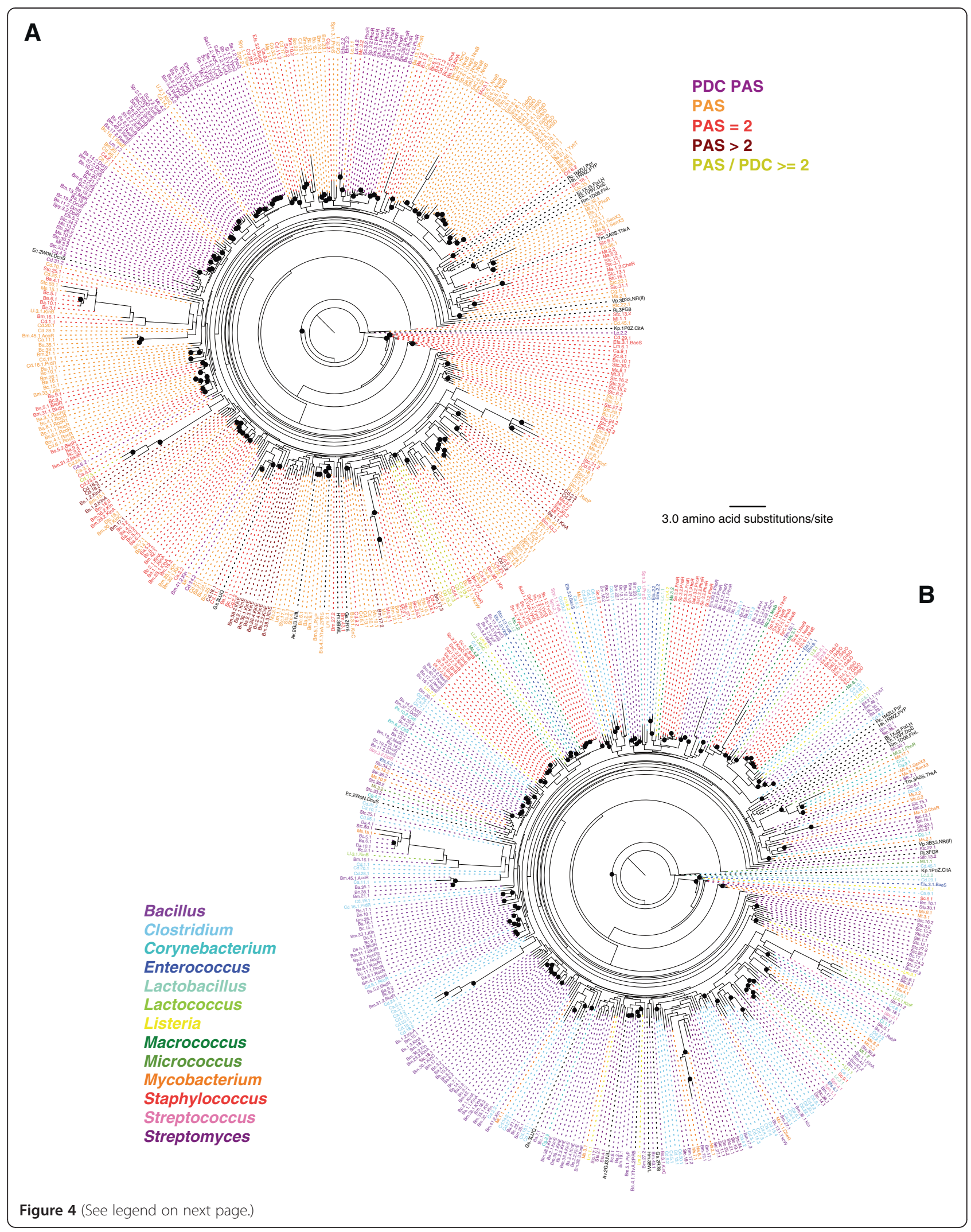


(See figure on previous page.)

Figure 4 Maximum likelihood phylogeny of PAS domain protein sequences. 372 PAS domain sequences obtained from 28 Gram-positive bacterial genomes are included. As references, the phylogeny is shown in two color schemes: based on PDC/PAS domain architectures (A) and based on bacterial genera (B). Black and green circles at internal nodes represent those supported by bootstrap values greater than $60 \%$ and those supported by all three phylogenetic methods although bootstrap values were 60\% or lower. Fourteen PAS domain sequences whose structures are known and used as the search queries were included in the phylogeny and they are labeled with black letters. One PDC domain sequence, 1P0Z (CitA, Klebsiella pneumoniae), was also included as the outgroup and is shown in black. See Table 1 and (Additional file 1: Table S1) and (Additional file 3: Table S2) for the species name abbreviations and protein IDs. Species abbreviations not listed in Table 1 are Av (Azotobacter vinelandii), Bj (Bradyrhizobium japonicum), Gs (Geobacter sulfurreducens), Hm (Haloarcula marismortui), Hh (Halorhodospira halophila), Rc (Rhodospirillum centenum), Rj (Rhodococcus jostii), Rm (Rhizobium meliloti), Tm (Thermotoga maritima), Vp (Vibrio parahaemolyticus).

that the duplication of these domains must have occurred prior to the divergence of these two species. Very different evolutionary relationships are found among multiple PAS domains identified in another kinase, KinA. The B. subtilis KinA has three PAS domains (shown in brown in Figure 4A) whereas the other Bacillus KinA proteins have only two PAS domains (shown in red). Only the first PAS domains of B. authracis (Ba.1.1), B. cereus (Bc.2.1), and B. megaterium (Bm.28.1), and the second PAS domains of $B$. authracis and B. cereus (Bc.2.2 and Ba.1.2) form clusters. While the second PAS domain of B. megaterium (Bm.28.2) and the second and third PAS domains of B. subtilis (Bs.1.2 and Bs.1.3) appear to share some similarity, the first PAS domain of B. subtilis (Bs.1.1) is highly diverged from any of these KinA PAS domains. This is interesting because this particular B. subtilis PAS domain (Bs.1.1) is involved in binding ATP and regulating the sporulation phosphorelay system [39]. Another interesting observation can be seen with two types of transcriptional regulators found in the Bacillus genomes, BkdR and RocR. BkdR proteins have two PAS domains (shown in red in Figure 4A) and RocR proteins have a single PAS domain (shown in orange in Figure 4A). The PAS domains of these proteins form a single cluster where the PAS domains of RocR are more similar to the second PAS domains of BkdR, illustrating a dynamic evolution of these PAS-containing transcriptional regulators. Specifically, after the duplication of PAS domains in the ancestral Bacillus BkdR proteins, the ancestral RocR protein was derived from the second PAS domain region of the BkdR protein. As both RocR and BkdR are involved in regulating amino acid catabolic pathways (arginine/ornithine and branched chain amino acids respectively) $[40,41]$, this evolution may be linked to their functions.

Compared to the PAS phylogeny, PDC domains are significantly more divergent as indicated by the much longer branch lengths in Figure 5. Reflecting their higher degree of divergence, the clustering patterns shown in the PDC phylogeny are much more complex. For example, PhoR and WalK ortholog proteins, both are clustered individually in the PAS phylogeny (Figure 4), but are found in dispersed locations in the PDC phylogeny (e.g., PDC domains from three Bacillus PhoR proteins: Bm.39.1 from B. megaterium, Bs.9.1 from B. subtilis, and
Bc14.1 from B. cereus, are completely separated). Some proteins we examined contain two PDC domains and they are shown as "Proximal" and "Distal" in Figure 5A (in light blue and in light purple, respectively). Interestingly, the majority of the "Proximal" PDC domains form a single large cluster indicating their monophyletic origin. On the other hand, the "Distal" PDC domains are more divergent and show evolutionary patterns distinct from the "Proximal" domain.

In order to understand how phylogenies obtained from PAS and PDC domains individually correlate with the entire protein evolution, we used the WalK ortholog proteins as an example. We reconstructed three phylogenies from 17 WalK proteins: from PAS domain, from PDC domain, and from the entire protein sequences (see Additional file 2: Figure S1). For this analysis, the WalK orthologs from Lactococcus lactis and Streptococcus sp. were excluded due to the absence of a PDC domain in the extracellular loop. The entire WalK proteins are approximately 610 amino acid (aa) length, while their PAS and PDC domains are between 88-147 aa. The entire WalK protein phylogeny shows three clearly supported clusters: the WalK proteins from Macrococcus caseolyticus and the Staphylococcus sp., those from Bacillus sp. and Listeria monocytogenes, and those from Lactobacillus casei and Enterococcus sp. As described before, PAS domains of WalK proteins are much more conserved than their PDC domains, and their phylogenetic clustering was completely congruent with the entire protein relationship. In striking contrast, with the exception of the Staphylococcus domains, no clear clustering pattern was found in the PDC phylogeny. Taken together, these data suggest that PAS domain evolution is more conserved and protein/function-specific, while PDC domains are highly divergent and possibly contributed to a more dynamic protein function evolution.

\section{PDC/PAS domains of staphylococci}

The medical importance of staphylococci and our interest in dicarboxylic acid sensing led us to examine the staphylococcal PDC/PAS proteins to gain insight into their potential function(s). As stated earlier, the number of PDC/PAS proteins correlates well with motility, such that motile bacteria have 4 times as many PDC/PAS proteins as do non-motile bacteria. The staphylococci are 


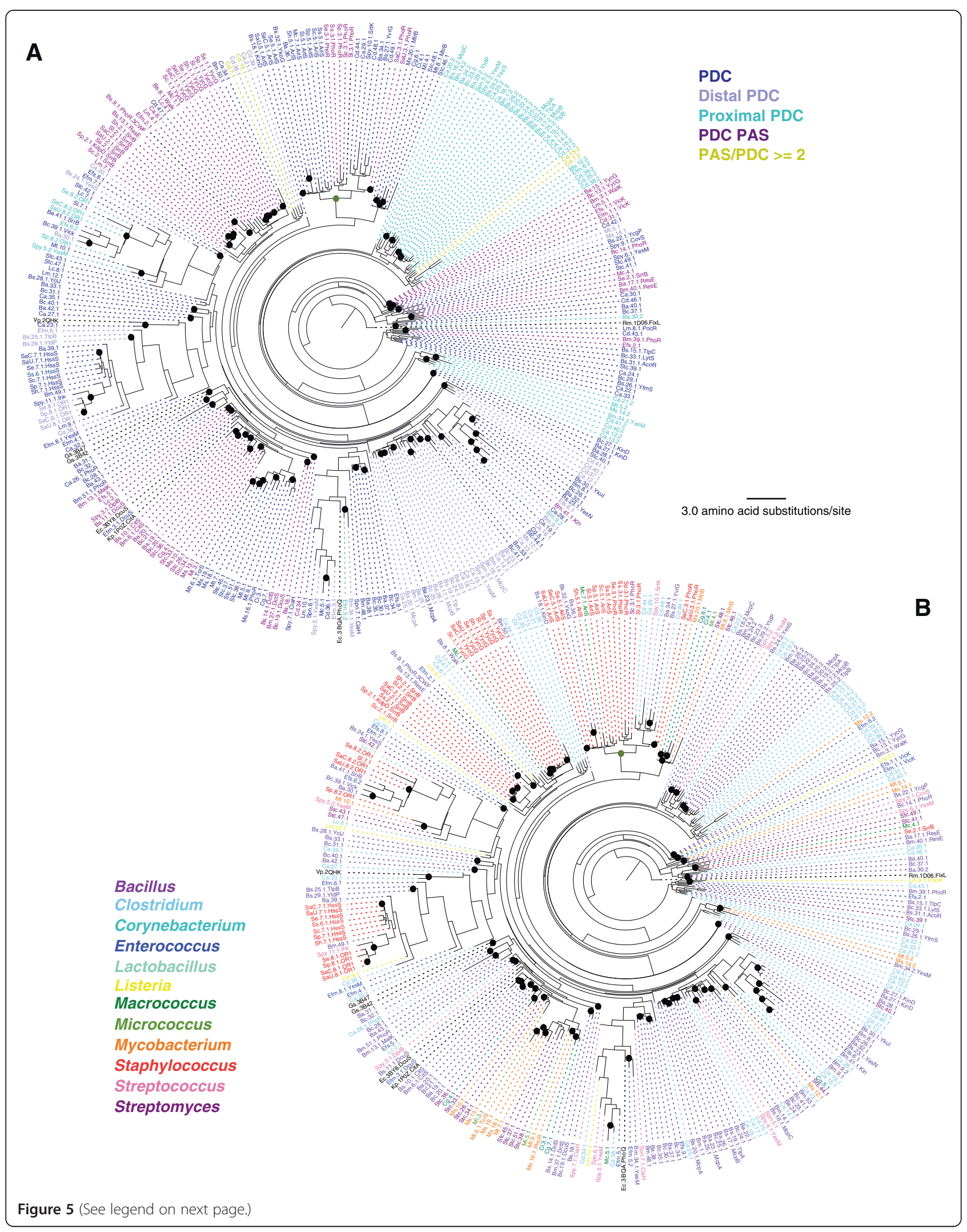


(See figure on previous page.)

Figure 5 Maximum likelihood phylogeny of PDC domain protein sequences. 303 PDC domain sequences identified from 28 Gram-positive bacterial genomes are included. The phylogeny is shown in two color schemes: based on PDC/PAS domain architectures (A) and based on bacterial genera (B). "Distal" and "Proximal" PDCs are the first and second, respectively, domains in the two-PDC proteins (shown as "PDC = 2" in Figure 3). Black and green circles at internal nodes represent those supported by bootstrap values greater than $60 \%$ and those supported by all three phylogenetic methods although bootstrap values were 60\% or lower. Seven PDC domain sequences whose structures are known and used as the search queries were included in the phylogeny and they are labeled with black letters. One PAS domain sequence, 1D06 (FixL, Rhizhobium meliloti), was included as the outgroup and is shown in black. See Table 1 and (Additional file 1: Table S1) and (Additional file 3: Table S2) for the species name abbreviations and protein IDs. Species abbreviations not listed in Table 1 are Gs (Geobacter sulfurreducens), Rm (Rhizobium meliloti), and Vp (Vibrio parahaemolyticus).

non-motile bacteria that are closely related to the motile bacterial species in the Bacillus genus (Figure 3). Based on the maximum likelihood phylogeny of the $16 \mathrm{~S}$ rDNA sequences in Figure 3, when the staphylococcaceae (i.e., Macrococcus and Staphylococcus) diverged from Bacillus megaterium it appears that motility was lost. Prior to that divergence, all of the related bacteria (e.g., Listeria monocytogenes and Bacillus sp.) were/are motile (B. anthracis is a notable exception). Interestingly, the Staphylococcus sp. are most closely related to Macrococcus caseolyticus, which is a non-pathogenic and non-motile bacterium found in milk and on animal skin, suggesting that the transition from free-living bacterium to being host-adapted coincided with the loss of motility. The genus Staphylococcus is comprised of species that have between 6 (i.e., S. saprophyticus) and 9 (i.e., S. carnosus) proteins having one or more PDC/PAS domains, while the Bacillus genus contains species having between 32 (i.e., B. subtilis) and 53 (i.e., B. megaterium) PDC/PAS proteins (Figure 3 and Table 1). The loss of PDC/PAS proteins by staphylococci is consistent with the transition from a motile life-style to a non-motile life-style; however, the loss of motility is unlikely to be the complete explanation for the evolutionary loss of PDC/ PAS proteins. A second phenotypic difference between the Staphylococcus and Bacillus genera is the ability to sporulate, with the Bacillus sp. able to form spores but not the staphylococci. Four of the kinases (i.e., KinA, $\mathrm{KinC}$, KinD, and KinE) that regulate sporulation in Bacillus sp. [42] all contain PDC/PAS domains (see Additional file 1: Table S1). In contrast to the Bacillus sp., staphylococci have an oxygen-sensing two-component system (NreB-NreC) that regulates transcription of the nitrate and nitrite reductase systems $[43,44]$. NreB, the sensor histidine kinase portion of the two component system, uses a PAS domain to coordinate binding of an oxygenlabile $[4 \mathrm{Fe}-4 \mathrm{~S}]^{2+}$ iron-sulfur cluster. Lastly, it was shown that the number of PDC/PAS proteins correlates with the presence of electron transport proteins [28]. Bacillus sp. and Staphylococcus sp. all possess active electron transport systems; hence, the difference in the number of PDC/PAS proteins is unlikely to be due to the changes in electron transport. In total, these observations suggest the transition from a free-living bacterium to a host-adapted bacterium coincided with the loss of most PDC/PAS proteins from the Staphylococcus genus through reductive evolution.

While reductive evolution likely led to the loss of many PDC/PAS proteins in staphylococci, all staphylococci in this study maintained orthologs of six PDC/PAS proteins; specifically, WalK (shown as yycG or VicK in Additional file 1: Table S1), SrrB, PhoR, ArlS, HssS, and GdpP. Five of these proteins are members of two component signal transduction systems that are important for oxygen-sensing (SrrB) [38], response to phosphate-limited conditions (PhoR) [45], regulation of hemin transport (HssS) [46], autolysis (ArlS) [47], and the regulation of cell wall biosynthesis (WalK) $[48,49]$. The sixth conserved PDC/PAS protein, GdpP, has recently been described as being a cyclic-di-AMP phosphodiesterase that suppresses a mutation in $S$. aureus lipoteichoic acid biosynthesis [50]. Importantly, these sensory proteins not only regulate physiologically essential processes, but they also influence staphylococcal virulence and antibiotic resistance $[34,38,46,51,52]$. Although these six proteins are conserved in staphylococci, the evolutionary divergence of the PDC domains can be seen in staphylococci where a weakly conserved PDC-like region was identified in the ortholog of PhoR from S. haemolyticus and the HssS of S. lugdunensis (YP_003470952.1) lacks the PDC domain.

As mentioned before, all staphylococci included in this study, with the exception of $S$. saprophyticus, have NreB orthologs (see Additional file 1: Table S1). Similarly, four additional PDC/PAS proteins were identified that are present in some Staphylococcus species and whose functions are unknown. S. aureus, S. epidermidis and S. pseudintermedius have a protein containing two PDC domains (i.e., SaC.8/YP_185101.1, SaU.8/YP_001574141.1, Se.8/ NP_763721.1, Sp.8/YP_004150417.1; listed as OR1 in Additional file 1: Table S1), S. lugdunensis has a PDC domain containing protein (i.e., Sl.7/ ADC86684.1), and S. carnosus has two PAS domain containing proteins (i.e., Sc.8/YP_002635456.1 and Sc.9/YP_002634788.1). The two PDC domains of the OR1 orthologs in $S$. aureus, $S$. epidermidis and $S$. pseudintermedius cluster in two separate clades: the "Distal" PDC domains are close to the PDC domains of Hss that is involved in regulating hemin transport, while the "Proximal" PDC domain forms a 
cluster that also contains the single PDC domain of Sl.7 (see Figure 5). To gain more insight into the potential functions of these proteins, the protein sequences were analyzed by Gene Ontology (GO) and BLASTP/PSI-BLAST similarity search. These analyses revealed both proteins as having similarities to histidine kinases with "two-component sensor activity" (GO:0000155) and containing typical domains of histidine kinases (i.e. HisKa and HATPase). The possibility that both proteins are sensory histidine kinases of two component systems is supported by the presence of putative cognate response regulators adjacent to the corresponding genes. Interestingly, the genes coding for the OR1 proteins and their cognate response regulators are predicted to be co-transcribed with an upstream gene that is predicted to code for $\mathrm{ABC}$ transporter iron-binding proteins. Taken together, the genomic organization and the close phylogenetic relation of the OR1 PDC domain with that of HssS, suggest that OR1 may function in regulating iron homeostasis.

GO analysis of the S. carnosus Sc.8/YP_002635456.1 (found in Additional file 1: Table S1) protein, which contains two PAS domains, suggests it has two-component system sensor kinase activity, with some of the top BLASTP/PSI-BLAST hits from Bacillus sp. being an ethanolamine two-component sensor histidine kinase. Consistent with this suggestion, there is a putative ethanolamine transporter (ORF Sca_2366) located adjacent to the genes coding for the putative two component system. The PAS domain of the second PDC/PAS protein of unknown function in S. carnosus (i.e., Sc.9/YP_002634788.1) belongs to a highly supported cluster including six other single-PAS containing proteins (Ll.1/YP_001032211.1, Ca.1/NP_34 8158.1, Lc.3/YP_805881.1, Spn.4/YP_816768.1, Efs.4/ NP_814954.1, and Spn.1/YP_815994.1; Figure 4). Two of the PAS domains in this cluster, Lc.3/YP_805881.1 and Spn.4/YP_816768.1, are annotated as flavoproteins having oxidoreductase activity. The top hits in PSI-BLAST for Sc.9/YP_002634788.1 all have a sensory box in the Cterminus that is predicted to be a hemerythrin-binding domain. Hemerythrin-binding proteins are non-heme oxygen-binding proteins that are found in marine invertebrates (e.g., brachiopods) and some bacteria. Interestingly, in Firmicutes, hemerythrin-binding proteins are primarily present in anaerobic bacteria, such as the Clostridia; thus, it may be that Sc.9/YP_002634788.1 is important for $S$. carnosus in the adaptation to an anaerobic environment.

\section{Conclusions}

Bacterial evolution has led to huge variations in the number of PDC/PAS proteins within all genera. Several hypotheses have been put forth to explain this variation as associated with pathogen status, genome size, or the presence of electron transport proteins. Undoubtedly, all of these bacterial traits contribute to variation in the number of PDC/PAS proteins; however, our analysis suggests that the number of PDC/PAS domains correlates to bacterial motility. This observation partially explains the selective loss of PDC/PAS domains in the genus Staphylococcus after its divergence from the genus Bacillus.

\section{Methods}

\section{Bacterial genomes}

48 bacterial genomes from the National Center for Biotechnology and Information (NCBI) [53] were chosen so as to include pathogenic and non-pathogenic bacteria, commensal bacteria, and saprophytes (Table 1). Of the 48 genomes, 32 are from Gram-positive bacteria belonging to the Firmicute and Actinobacteria phyla and representing 14 genera (Clostridium, Streptococcus, Enterococcus, Listeria, Lactobacillus, Lactococcus, Bacillus, Staphylococcus, Mycobacterium, Corynebacterium, Micrococcus, Streptomyces, Macrococcus, and Bifidobacterium). For the detailed study of staphylococci, the genomes of seven different species and two strains of Staphylococcus aureus subsp. aureus (i.e., COL and USA300 TCH1516) were selected. In addition, sixteen Gram-negative bacterial genomes representing Alpha, Gamma, and Epsilon proteobacteria, two Spirochaetes (Leptospira and Treponema), and two Cyanobacteria (Synechococcus and Nostoc) were included in the analysis.

\section{PDC/PAS domain search methods}

Due to the extreme divergence $(<20 \%$ identity) of PDC/ PAS domain sequences, sequence similarity search methods such as Basic Local Alignment Search Tool (BLAST) $[23,54]$ or FASTA [55] are minimally effective at identifying PDC/PAS proteins. Profile or profile HMM methods such as PSI-BLAST [23] and HMMER [56] have been more successful at identifying PDC/PAS proteins (e.g., [24]). More recently, HMM-to-HMM comparisons were used by Chang et al. [19] to identify PDC domains in Bacillus subtilis. In order to increase the likelihood of identifying PDC/PAS proteins as thoroughly as possible, we chose to use HHblits (HH-suite 2.0.14) [26], the current state-of -the-art method. Although HHblits is in general highly sensitive, due to its use of prefilters and discretization of database HMMs [26], occasionally some sequences can be missed by HHblits. In order to complement the results of HHblits, we also used HHsearch (ver. 1.5.1) [57]. Both packages were downloaded [58] and installed on a local Linux server. The methods require profile HMMs constructed from queries (PDC/PAS domain sequences) as well as from protein sequences included in the bacterial genome databases. Secondary structure information of each sequence was also added to the HMMs. Additional details of the search methods are described in the following sections. 


\section{PDC/PAS domain queries}

3D-structural information from 86 PDC/PAS domain sequences (60 PAS and 26 PDC domains; see in Additional file 3: Table S2) were downloaded from the Protein Data Bank (PDB) $[59,60]$. These structures represented eight bacterial phyla that contained proteins with diverse ligand affinities. When proteins have multiple domains, PAS or PDC domains were extracted and used as queries individually.

\section{Preparation of HMM queries and HMM databases}

In order to perform HMM-to-HMM comparisons, both query sequences and protein sequences in each bacterial genome needed to be converted to HMMs. For HHblits, this was done by using the programs hhblits, addss.pl, and hhmake, all using the default settings. The program hhblits was used to iteratively search the non-redundant database (nr20, included in the $\mathrm{HH}$-suite 2.0.14) with a protein sequence from the queries or from the bacterial genomes. The options were set as default, except to perform three iterations with the E-value threshold for inclusion at 0.001. From each iteration, sequences within the inclusion threshold were added to the query or to its multiple sequence alignment (MSA). The script addss.pl was then used to incorporate the secondary structure information predicted by PSIPRED (ver. 3.2) [61] to the MSA generated for each protein sequence. Finally, the program hhmake was used to generate the HMM from each MSA. To generate the final HHblits databases for the bacterial genome data sets, the program hhblitsdb.pl was also used. For HHsearch, the overall process taken was similar to what described for HHblits. In order to build MSAs for query sequences, the program builali.pl was used, which runs PSI-BLAST (ver. 2.2.17; inclusion E-value threshold $=0.001$ and 8 iterations) against the filtered NCBI non-redundant protein database obtained from HHsearch 1.5.1.

\section{PDC/PAS domain sequence search against bacterial genomes}

The PDC/PAS domain sequence search was done with hhblits and hhsearch programs using each domain HMM query against each bacterial HMM database prepared above. The same settings were used for iteration numbers and inclusion E-value thresholds. For both HHblits and HHsearch, the hits were considered as significant based on the E-value threshold of 1, 95\% probability (this is "the probability of template to be a true positive" calculated by HHblits), and the length of aligned HMM regions to be 90 amino acids. Since known PDC/PAS domains are 100140 amino acid long (see Additional file 3: Table S2), 90 amino acids cover $65-90 \%$ of the domain. This alignment coverage gave us sufficiently strong search results. When
HHblits did not give a significant hit but HHsearch did, the hit by HHsearch was also included. In some cases, we also found significant hits shorter than 90 amino acids whose E-values and probabilities were within our threshold. We examined these cases for a) their alignment quality, b) if PAS/PDC topology is recognized from the entire protein, and c) if their orthologs in other species have a PAS/PDC domain. We identified 11 cases to be positive based on these further analyses. These HHsearch-based as well as short PAS/PDC domains are marked in (Additional file 1: Table S1). All bacterial protein HMM databases and query HMMs used for this study are available from: http://bioinfolab.unl.edu/emlab/PAS_PDC.

\section{Classification of PDC/PAS domains}

All the significant PDC/PAS domains identified from HHblits and HHsearch were classified based on their significant similarities with either PAS or PDC query. As described before, PAS and PDC domains are defined, respectively, with $\alpha-2 \beta-4 \alpha-3 \beta$ and $3 \alpha-2 \beta-1 / 2 \alpha-3 \beta-\alpha$ topologies (see the examples in Additional file 4: Figures S2 and S3). All our identified hits within the threshold followed these secondary structure patterns. Note that GAF domains (named for their presence in cyclic GMP-specific phosphodiesterase, adenylyl cyclase, and FhIA), for example, share a similar folding pattern $(\alpha-3 \beta-\alpha-\beta-\alpha-2 \beta-\alpha)$; however, using a conservative threshold with $\mathrm{HHblits} / \mathrm{HH}$ search, our results do not include GAF-domain containing proteins. All identified PAS and PDC domains are listed in (Additional file 1: Table S1). For convenience, we labeled all PDC/PAS proteins using abbreviations of species name and numbers (e.g., Cd.1 for the first protein of Clostridium difficile). All PDC and PAS domains of each protein are then numbered in the order of their occurrence (e.g., Cd.1.1 and Cd.1.2 for the two PAS domains identified in the protein Cd.1). These domain names are used in all alignments and phylogenies.

\section{Multiple sequence alignment}

All identified PAS and PDC domains were individually aligned using PRALINE [62]. BLOSUM45 amino acid substitution matrix, open and extension gap penalties of 12 and 1 , respectively, PSI-BLAST against the protein non-redundant database with 3 iterations and E-value threshold $=0.01$, DSSP structural features, and PSIPRED secondary structure prediction were chosen from the options. The MSAs of all identified PAS and PDC domains with their predicted secondary structures are available from: http://bioinfolab.unl.edu/emlab/PAS_PDC.

For bacterial $16 \mathrm{~S}$ ribosomal DNA (rDNA) sequences, the alignment was generated using MAFFT v6.847b [63] with the L-INS-i algorithm. This MSA is also available from our website. 


\section{Phylogenetic tree reconstruction}

The maximum-likelihood phylogenies for the PAS and PDC domains were reconstructed using RAxML version 7.0.4 [64]. All the options were set to default except for PROTGAMMAJTT for the $-\mathrm{m}$ option, which estimates the gamma model for rate heterogeneity and uses the JTT amino acid substitution model. We also used two other phylogenetic methods, FastTree (ver. 2.1.4) [65] and Neighbor-Joining (PHYLIP 3.69) [66]. For both methods, the options for the gamma model of rate heterogeneity and the JTT amino acid substitution model were used. Bootstrap analysis was done with 1000 pseudoreplicates for all phylogenetic analyses. The program consense from the PHYLIP package was used to estimate the consensus phylogeny as well as the bootstrap values. In addition to bootstrap supporting values, we also calculated the supporting values for internal branches by three phylogenetic methods ( 3 as the highest support). For the $16 \mathrm{~S}$ rDNA phylogeny, the maximum-likelihood phylogeny was reconstructed using PhyML version 3.0 [67]. All options were set to default except for estimating the gamma distribution parameter. Bootstrap analysis was done with 1000 pseudoreplicates.

\section{Ortholog and gene ontology analysis of Staphylococcus proteins}

Orthology of all PDC/PAS proteins identified from Staphylococcus genomes was determined using reciprocal best-hit analysis in a BLAST similarity search. In this analysis, an unknown PDC/PAS protein from a Staphylococcus species was first used as a query for a protein similarity search using the blastp program against the $S$. aureus COL genome. The top hit protein in S. aureus was then used as the blastp search query against the original Staphylococcus genome. If the top hit from this second search was the original query protein, the query protein in the original species and the $S$. aureus protein are bidirectional or reciprocal best hits and considered to be coded by orthologous genes. When the $S$. aureus ortholog was not identified as a known gene, reciprocal blast analysis was performed also using other Staphylococcus genomes. Besides orthology analysis, functions of Staphylococcus PDC/PAS proteins were also inferred by BLAST similarity search against the Gene Ontology database using AmiGo $[68,69]$.

\section{Statistical analyses}

All statistical analyses were done using JMP v10.0.2 (SAS Institute Inc.). Our dataset included eight genomes of closely related Staphylococcus species; hence, there was a possibility that over-representation of staphylococcal genomes could skew our data analysis. To address this possibility, all statistical analyses were done twice; once using the entire data set from all 48 bacterial genomes, and once using the data from only 43 bacterial genomes, which had only three representatives from the eight Staphylococcus genomes: S. aureus TCH1516, S. saprophyticus, and S. carnosus.

\section{Additional files}

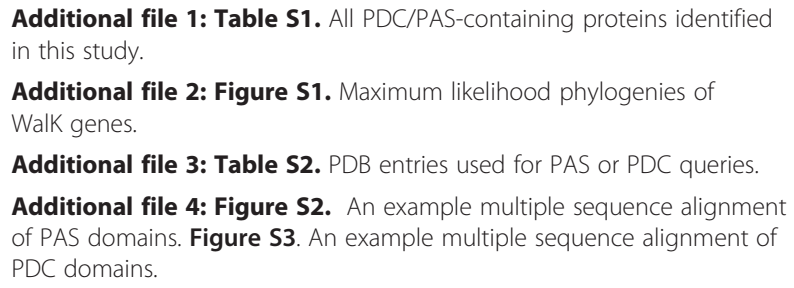

Additional file 2: Figure S1. Maximum likelihood phylogenies of WalK genes.

Additional file 3: Table S2. PDB entries used for PAS or PDC queries. Additional file 4: Figure S2. An example multiple sequence alignment of PAS domains. Figure S3. An example multiple sequence alignment of PDC domains.

\section{Competing interests}

The authors declare that they have no competing interests.

\section{Authors' contributions}

RG and GAS conceived of the project. NS, RG, ENM, HM, and GAS contributed to the experimental design and execution. ENM and KME performed the statistical analyses. NS, RG, ENM, HM, KME, and GAS wrote the manuscript. All authors read and approved the final manuscript.

\section{Acknowledgements}

GAS was supported by funds provided through the Hatch Act to the University of Nebraska Institute of Agriculture and Natural Resources and by funds provided through the NIH (Al087668). This work was completed utilizing the Holland Computing Center of the University of Nebraska. We would like to thank the reviewers for their helpful suggestions on improving our manuscript.

\section{Author details}

${ }^{1}$ Department of Computer Science and Engineering, University of Nebraska, Lincoln, NE 68588-0115, USA. ${ }^{2}$ School of Veterinary Medicine and Biomedical Sciences, University of Nebraska, Lincoln, NE 68583-0905, USA. ${ }^{3}$ School of Biological Sciences, University of Nebraska, Lincoln, NE 68588-0118, USA. ${ }^{4}$ Department of Statistics, University of Nebraska, Lincoln, NE 68583-0963, USA. ${ }^{5}$ School of Biological Sciences and Center for Plant Science Innovation, University of Nebraska, Lincoln, NE 68588-0118, USA.

Received: 17 December 2012 Accepted: 25 July 2013

Published: 31 July 2013

\section{References}

1. Anantharaman V, Koonin EV, Aravind L: Regulatory potential, phyletic distribution and evolution of ancient, intracellular small-molecule-binding domains. J Mol Biol 2001, 307(5):1271-1292.

2. Galperin MY: Bacterial signal transduction network in a genomic perspective. Environ Microbiol 2004, 6(6):552-567.

3. Ponting CP, Aravind L: PAS: a multifunctional domain family comes to light. Curr Biol 1997, 7(11):R674-677.

4. Taylor BL, Zhulin IB: PAS domains: internal sensors of oxygen, redox potential, and light. Microbiol Mol Biol Rev 1999, 63(2):479-506.

5. Zhulin IB, Taylor BL, Dixon R: PAS domain S-boxes in Archaea, Bacteria and sensors for oxygen and redox. Trends Biochem Sci 1997, 22(9):331-333.

6. An S, Wu J, Zhang LH: Modulation of Pseudomonas aeruginosa biofilm dispersal by a cyclic-Di-GMP phosphodiesterase with a putative hypoxiasensing domain. Appl Environ Microbiol 2010, 76(24):8160-8173.

7. Huang B, Whitchurch CB, Mattick JS: FimX, a multidomain protein connecting environmental signals to twitching motility in Pseudomonas aeruginosa. J Bacterio/ 2003, 185(24):7068-7076.

8. Morgan R, Kohn S, Hwang SH, Hassett DJ, Sauer K: BdIA, a chemotaxis regulator essential for biofilm dispersion in Pseudomonas aeruginosa. J Bacteriol 2006, 188(21):7335-7343. 
9. Parish T, Smith DA, Roberts G, Betts J, Stoker NG: The senX3-regX3 twocomponent regulatory system of Mycobacterium tuberculosis is required for virulence. Microbiology 2003, 149(Pt 6):1423-1435.

10. Rickman L, Saldanha JW, Hunt DM, Hoar DN, Colston MJ, Millar JB, Buxton RS: A two-component signal transduction system with a PAS domaincontaining sensor is required for virulence of Mycobacterium tuberculosis in mice. Biochem Biophys Res Commun 2004, 314(1):259-267.

11. Finn RD, Mistry J, Tate J, Coggill P, Heger A, Pollington JE, Gavin OL, Gunasekaran P, Ceric G, Forslund K, et al: The Pfam protein families database. Nucleic acids research 2010, 38(Database issue):D211-222.

12. Henry JT, Crosson S: Ligand-binding PAS domains in a genomic, cellular, and structural context. Annual review of microbiology 2011, 65:261-286.

13. Möglich A, Ayers RA, Moffat K: Structure and signaling mechanism of PerARNT-Sim domains. Structure 2009, 17(10):1282-1294.

14. Janausch IG, Zientz E, Tran QH, Kroger A, Unden G: C4-dicarboxylate carriers and sensors in bacteria. Biochim Biophys Acta 2002, 1553(1-2):39-56.

15. Somerville GA, Proctor RA: At the crossroads of bacterial metabolism and virulence factor synthesis in Staphylococci. Microbiol Mol Biol Rev 2009, 73(2):233-248

16. Götz F, Bannerman T, Schleifer KH: The Genera Staphylococcus and Macrococcus. In The Procaryotes, Volume 4. Edited by Dworkin M, Falkow S, Rosenberg E, Schleifer KH, Stackebrandt E. New York: Springer; 2006:5-75.

17. Hefti MH, Françoijs KJ, de Vries SC, Dixon R, Vervoort J: The PAS fold. A redefinition of the PAS domain based upon structural prediction. Eur J Biochem 2004, 271(6):1198-1208.

18. Scheu PD, Kim OB, Griesinger C, Unden G: Sensing by the membranebound sensor kinase DcuS: exogenous versus endogenous sensing of $C$ (4)-dicarboxylates in bacteria. Future Microbiol 2010, 5(9):1383-1402.

19. Chang C, Tesar C, Gu M, Babnigg G, Joachimiak A, Pokkuluri PR, Szurmant H, Schiffer M: Extracytoplasmic PAS-like domains are common in signal transduction proteins. J Bacteriol 2010, 192(4):1156-1159.

20. Cheung J, Hendrickson WA: Crystal structures of $\mathrm{C}_{4}$-dicarboxylate ligand complexes with sensor domains of histidine kinases DcuS and DctB. The Journal of biological chemistry 2008, 283(44):30256-30265.

21. Miyatake H, Mukai M, Park SY, Adachi S, Tamura K, Nakamura H, Nakamura K, Tsuchiya T, lizuka T, Shiro Y: Sensory mechanism of oxygen sensor FixL from Rhizobium meliloti: crystallographic, mutagenesis and resonance Raman spectroscopic studies. J Mol Biol 2000, 301(2):415-431.

22. Reinelt S, Hofmann E, Gerharz T, Bott M, Madden DR: The structure of the periplasmic ligand-binding domain of the sensor kinase CitA reveals the first extracellular PAS domain. J Biol Chem 2003, 278(40):39189-39196.

23. Altschul SF, Madden TL, Schäffer AA, Zhang J, Zhang Z, Miller W, Lipman DJ: Gapped BLAST and PSI-BLAST: a new generation of protein database search programs. Nucleic acids research 1997, 25(17):3389-3402.

24. Narikawa R, Okamoto S, Ikeuchi M, Ohmori M: Molecular evolution of PAS domain-containing proteins of filamentous cyanobacteria through domain shuffling and domain duplication. DNA Res 2004, 11(2):69-81.

25. Jaroszewski L, Rychlewski L, Li Z, Li W, Godzik A: FFAS03: a server for profile--profile sequence alignments. Nucleic Acids Res 2005, 33(Web Server issue):W284-288

26. Remmert M, Biegert A, Hauser A, Söding J: HHblits: lightning-fast iterative protein sequence searching by HMM-HMM alignment. Nature Methods 2012, 9(2):173-175.

27. Kondoh H, Ball CB, Adler J: Identification of a methyl-accepting chemotaxis protein for the ribose and galactose chemoreceptors of Escherichia coli. Proc Natl Acad Sci U S A 1979, 76(1):260-264.

28. Zhulin IB, Taylor BL: Correlation of PAS domains with electron transportassociated proteins in completely sequenced microbial genomes. Mol Microbiol 1998, 29(6):1522-1523.

29. Huycke MM, Sahm DF, Gilmore MS: Multiple-drug resistant enterococci: the nature of the problem and an agenda for the future. Emerg Infect Dis 1998, 4(2):239-249

30. Moran GJ, Krishnadasan A, Gorwitz RJ, Fosheim GE, McDougal LK, Carey RB, Talan DA: Methicillin-resistant $S$. aureus infections among patients in the emergency department. New Engl J Med 2006, 355(7):666-674.

31. Pfoh E, Wessels MR, Goldmann D, Lee GM: Burden and economic cost of group A streptococcal pharyngitis. Pediatrics 2008, 121(2):229-234.

32. Dubrac S, Bisicchia P, Devine KM, Msadek T: A matter of life and death: cell wall homeostasis and the WalKR (YycGF) essential signal transduction pathway. Mol Microbiol 2008, 70(6):1307-1322.
33. Friedman L, Alder JD, Silverman JA: Genetic changes that correlate with reduced susceptibility to daptomycin in Staphylococcus aureus. Antimicrob Agents Chemother 2006, 50(6):2137-2145.

34. Howden BP, McEvoy CR, Allen DL, Chua K, Gao W, Harrison PF, Bell J, Coombs G, Bennett-Wood V, Porter JL, et al: Evolution of multidrug resistance during Staphylococcus aureus infection involves mutation of the essential two component regulator WaIKR. PLoS pathogens 2011, 7(11):e1002359.

35. Szurmant H, Bu L, Brooks CL 3rd, Hoch JA: An essential sensor histidine kinase controlled by transmembrane helix interactions with its auxiliary proteins. Proc Natl Acad Sci U S A 2008, 105(15):5891-5896.

36. Nakano MM, Zuber P, Glaser P, Danchin A, Hulett FM: Two-component regulatory proteins ResD-ResE are required for transcriptional activation of fnr upon oxygen limitation in Bacillus subtilis. J Bacterio/ 1996, 178(13):3796-3802

37. Sun G, Sharkova E, Chesnut R, Birkey S, Duggan MF, Sorokin A, Pujic $P$, Ehrlich SD, Hulett FM: Regulators of aerobic and anaerobic respiration in Bacillus subtilis. J Bacteriol 1996, 178(5):1374-1385.

38. Yarwood JM, McCormick JK, Schlievert PM: Identification of a novel twocomponent regulatory system that acts in global regulation of virulence factors of Staphylococcus aureus. J Bacteriol 2001, 183(4):1113-1123.

39. Stephenson K, Hoch JA: PAS-A domain of phosphorelay sensor kinase A: a catalytic ATP-binding domain involved in the initiation of development in Bacillus subtilis. Proc Natl Acad Sci U S A 2001 98(26):15251-15256

40. Calogero S, Gardan R, Glaser P, Schweizer J, Rapoport G, Débarbouillé M: RocR, a novel regulatory protein controlling arginine utilization in Bacillus subtilis, belongs to the NtrC/NifA family of transcriptional activators. J Bacteriol 1994, 176(5):1234-1241.

41. Débarbouillé M, Gardan R, Arnaud M, Rapoport G: Role of BkdR, a transcriptional activator of the SigL-dependent isoleucine and valine degradation pathway in Bacillus subtilis. J Bacteriol 1999, 181(7):2059-2066.

42. Jiang M, Shao W, Perego M, Hoch JA: Multiple histidine kinases regulate entry into stationary phase and sporulation in Bacillus subtilis. Mol Microbiol 2000, 38(3):535-542.

43. Fedtke I, Kamps A, Krismer B, Götz F: The nitrate reductase and nitrite reductase operons and the narT gene of Staphylococcus carnosus are positively controlled by the novel two-component system NreBC. J Bacteriol 2002, 184(23):6624-6634.

44. Reinhart F, Huber A, Thiele R, Unden G: Response of the oxygen sensor NreB to air in vivo: Fe-S-containing NreB and apo-NreB in aerobically and anaerobically growing Staphylococcus carnosus. J Bacteriol 2010, 192(1):86-93.

45. Hulett FM: The Pho regulon. Washington, D.C.: ASM Press; 2002.

46. Torres VJ, Stauff DL, Pishchany G, Bezbradica JS, Gordy LE, Iturregui J Anderson KL, Dunman PM, Joyce S, Skaar EP: A Staphylococcus aureus regulatory system that responds to host heme and modulates virulence. Cell Host Microbe 2007, 1(2):109-119.

47. Fournier B, Hooper DC: A new two-component regulatory system involved in adhesion, autolysis, and extracellular proteolytic activity of Staphylococcus aureus. J Bacteriol 2000, 182(14):3955-3964

48. Dubrac S, Boneca IG, Poupel O, Msadek T: New insights into the WalK/ WalR (YycG/YycF) essential signal transduction pathway reveal a major role in controlling cell wall metabolism and biofilm formation in Staphylococcus aureus. J Bacteriol 2007, 189(22):8257-8269.

49. Dubrac $S$, Msadek T: Identification of genes controlled by the essential YycG/YycF two-component system of Staphylococcus aureus. J Bacteriol 2004, 186(4):1175-1181.

50. Corrigan RM, Abbott JC, Burhenne H, Kaever V, Gründling A: c-di-AMP is a new second messenger in Staphylococcus aureus with a role in controlling cell size and envelope stress. PLoS Pathogens 2011, 7(9):e1002217.

51. Burnside K, Lembo A, de Los RM, lliuk A, Binhtran NT, Connelly JE, Lin WJ, Schmidt BZ, Richardson AR, Fang FC, et al: Regulation of hemolysin expression and virulence of Staphylococcus aureus by a serine/threonine kinase and phosphatase. PloS one 2010, 5(6):e11071.

52. Fournier B, Klier A, Rapoport G: The two-component system ArlS-ArlR is a regulator of virulence gene expression in Staphylococcus aureus. Mol Microbiol 2001, 41(1):247-261.

53. National Center for Biotechnology and Information (NCBI). http://www. ncbi.nlm.nih.gov. 
54. Altschul SF, Gish W, Miller W, Myers EW, Lipman DJ: Basic local alignment search tool. J Mol Biol 1990, 215(3):403-410.

55. Pearson WR, Lipman DJ: Improved tools for biological sequence comparison. P Natl Acad Sci USA 1988, 85(8):2444-2448.

56. Eddy SR: Profile hidden Markov models. Bioinformatics 1998, 14(9):755-763.

57. Söding J: Protein homology detection by HMM-HMM comparison. Bioinformatics 2005, 21(7):951-960.

58. HH-suite distribution server. ftp://toolkit.genzentrum.lmu.de.

59. Berman H, Henrick K, Nakamura H: Announcing the worldwide Protein Data Bank. Nat Struct Biol 2003, 10(12):980.

60. Protein Data Bank. http://www.rcsb.org/pdb.

61. Jones DT: Protein secondary structure prediction based on positionspecific scoring matrices. J Mol Biol 1999, 292(2):195-202.

62. Simossis VA, Heringa J: PRALINE: a multiple sequence alignment toolbox that integrates homology-extended and secondary structure information. Nucleic Acids Res 2005, 33(Web Server issue):W289-294.

63. Katoh K, Misawa K, Kuma K, Miyata T: MAFFT: a novel method for rapid multiple sequence alignment based on fast Fourier transform. Nucleic Acids Res 2002, 30(14):3059-3066.

64. Stamatakis A: RAxML-VI-HPC: maximum likelihood-based phylogenetic analyses with thousands of taxa and mixed models. Bioinformatics 2006, 22(21):2688-2690.

65. Price MN, Dehal PS, Arkin AP: FastTree: computing large minimum evolution trees with profiles instead of a distance matrix. Mol Biol Evol 2009, 26(7):1641-1650.

66. Felsenstein J: PHYLIP (Phylogeny Inference Package) version 3.6. In Distributed by the author. Seattle: Department of Genome Sciences, University of Washington; 2005

67. Guindon S, Dufayard JF, Lefort V, Anisimova M, Hordijk W, Gascuel O: New algorithms and methods to estimate maximum-likelihood phylogenies: assessing the performance of PhyML 3.0. Syst Biol 2010, 59(3):307-321.

68. Carbon S, Ireland A, Mungall CJ, Shu S, Marshall B, Lewis S: AmiGO: online access to ontology and annotation data. Bioinformatics 2009, 25(2):288-289.

69. AmiGO. http://amigo.geneontology.org/cgi-bin/amigo/go.cgi.

doi:10.1186/1471-2164-14-524

Cite this article as: Shah et al.: Reductive evolution and the loss of PDC/ PAS domains from the genus Staphylococcus. BMC Genomics 2013 14:524.

\section{Submit your next manuscript to BioMed Central and take full advantage of:}

- Convenient online submission

- Thorough peer review

- No space constraints or color figure charges

- Immediate publication on acceptance

- Inclusion in PubMed, CAS, Scopus and Google Scholar

- Research which is freely available for redistribution 